DOI: $10.24850 / \mathrm{j}$-tyca-2019-02-10

Notas

\title{
Experimentos de equilibrio de lechos fluviales de arena y grava
}

Flume experiments on the equilibrium of sand-gravel bed rivers

Juan P. Martín-Vide ${ }^{1}$

Francisco Núñez-González ${ }^{2}$

${ }^{1}$ Universitat Politècnica de Catalunya-BarcelonaTech, Barcelona, España, juan.pedro.martin@upc.edu

${ }^{2}$ Leichtwei $\beta$-Institut für Wasserbau, Technische Univ. Braunschweig, Braunschweig, Alemania, fngon@yahoo.com

Autor para correspondencia: Juan P. Martín-Vide, juan.pedro.martin@upc.edu

\section{Resumen}

Se describe una instalación de laboratorio que permite realizar ensayos en canal con alimentación separada de arena y grava, como es caracterísitico de los lechos de muchos ríos. Se hacen ensayos de gran duración, en que el transporte como carga de fondo llega a valer $1 \mathrm{~kg} / \mathrm{s}$. El material sólido que sale del canal se envía hacia la cabecera, en donde se separa la grava de la arena. La alimentación se hace con dos tolvas, una para cada material, cuyos pesos se miden continuamente para conocer los caudales de alimentación. Los ensayos persiguen alcanzar el equilibrio, es decir, la permanencia del lecho sin erosión ni crecimiento a largo plazo. Se ofrecen ejemplos reales de varios tipos de ensayo: recirculación con erosión del lecho y con crecimiento del lecho, 
recirculación pura y alimentación pura. Los cuatro son ejemplos de transporte sólido con movilidad igualada. Se presenta una discusión del significado de los ensayos.

Palabras clave: canal hidráulico, lecho de arena y grava, transporte de sedimento, recirculación vs. alimentación, equilibrio fluvial, hidráulica fluvial, incisión, erosión, acumulación.

\section{Abstract}

A laboratory facility which is able to perform hydraulic tests with the separate feeding of sand and gravel into a flume is described. Mixtures of sand and gravel are tipically found in many river beds. The facility allows for long duration tests with bed load rates as high as $1 \mathrm{~kg} / \mathrm{s}$. The material collected in the flume outlet is sent to the inlet area where gravel fractions are separated from sand. The feeding of a test is made by two hoppers, one for each material. Each hopper weight is continuously monitored to know the feeding rate (bed load rate). All the tests search for equilibrium, that is to say for a steady bed profile with neither incision nor accumulation in the long term. Different types of tests are shown as examples: recirculation with bed incision and accumulation, pure recirculation and pure feeding. The four are examples of equal mobility in terms of sediment transport. A discusssion follows about the meaning of flume tests.

Keywords: Flume, sand-gravel mixtures, sediment transport, recirculation vs. Feeding, river equilibrium, fluvial hydraulics, incision, degradation, aggradation.

Recibido: 25/02/2016

Aceptado: 18/07/2018

\section{Introducción}


El flujo de agua en un río aluvial puede mover las partículas que forman su lecho y transportarlas de varias maneras, por ejemplo, saltando por el fondo. Este transporte de sólidos origina cambios del propio lecho. Mirando de cerca, los dos fenómenos más importantes son: 1) las formas de fondo, es decir las ondulaciones del lecho, y 2) el acorazamiento, es decir que las partículas más gruesas situadas en la superficie protejan a las más finas. Mirando de lejos, el transporte sólido puede afectar al perfil longitudinal del río. Las alternativas al respecto son la erosión del fondo (descenso de su nivel o incisión) y, su opuesto, la acreción o crecimiento del nivel de fondo. Ambos pueden venir acompañados de un cambio de pendiente.

La noción de equilibrio fluvial se refiere a que, a largo plazo y haciendo abstracción de los fenómenos descritos "mirando de cerca", el perfil longitudinal del río ni asciende ni desciende (ni cambia de pendiente) a pesar del transporte sólido de material de fondo; en otras palabras, el lecho móvil o "vivo" permanece estable "mirándolo de lejos". El tipo de transporte que se menciona no incluye la suspensión de partículas finas con origen en la cuenca, sino sólo al material con origen en el fondo.

El estudio del equilibrio ha sido una pieza clave de la investigación en hidráulica fluvial desde sus inicios. Por ejemplo, el transporte sólido de fondo se determina exigiendo el equilibrio. De lo contrario, el caudal de partículas transportadas estaría desvirtuado por la contribución de las que son arrancadas del fondo si el perfil está al mismo tiempo descendiendo o, inversamente, desvirtuado por la sustracción de las que están depositándose en el fondo si al mismo tiempo está ascendiendo. Las fórmulas de transporte sólido, que dan un valor del caudal sólido de fondo como función del flujo de agua, suponen la existencia de equilibrio. En ello está implícito, por cierto, que exista una aportación de sólidos desde aguas arriba suficiente como para colmar la capacidad de transporte (dada por las fórmulas), de manera que la fórmula exprese el transporte verdadero. Para el equilibrio es también necesario un régimen uniforme, pues en régimen variado hay una variación de la capacidad de transporte según la posición, lo cual es incompatible con la estabilidad del perfil con el paso del tiempo. 
Este artículo trata de cómo se exige el equilibrio y cómo se alcanza en ensayos de laboratorio en lecho móvil. Esto se limita a un típico canal recto de laboratorio. El contexto es el de los ríos de gravas, cuyo estudio se ha apoyado en experimentos (Parker, 2008). El núcleo del artículo es la discusión de cómo trabaja una instalación proyectada para estudiar lechos aluviales de arena y grava en ríos de gran pendiente y, por tanto, con mucho transporte sólido, alargando la duración de ensayo para alcanzar el equilibrio. Por este motivo, la instalación debe manejar volúmenes muy grandes de sólidos. El objetivo del artículo es mostrar distintos tipos de ensayo de lecho móvil en equilibrio a través de la experiencia con dicha instalación $y$, en segundo lugar, discutir su significado para estudiar los perfiles de equilibrio de ríos reales.

\section{Descripción de la instalación}

La dificultad al planear ensayos de equilibrio en un canal de laboratorio es el gran volumen de material granular necesario. El equilibrio se alcanza poco a poco, con ritmo más lento cuanto más cerca se encuentre el estado presente del equilibrio. Esta propiedad se resume en la noción matemática de asíntota: el equilibrio es la asíntota cuando el tiempo tiende a infinito. En la práctica, el equilibrio se da por alcanzado si se consigue una cierta aproximación a la asíntota. Durante el tiempo transcurrido hasta el equilibrio práctico ha circulado agua y material sólido. El agua regresa a la cabecera del canal y se suele ignorar, por ello, el enorme volumen que ha circulado. Tal enormidad salta a la vista en el caso del caudal sólido, aunque sea mucho menor que el del agua: por ejemplo, $1 \mathrm{~kg} / \mathrm{s}$ de arena (alcanzado en esta instalación) en un ensayo de 10 horas de duración implica 36 toneladas de arena (unos $20 \mathrm{~m}^{3}$ ). Por ello, se ha de aplicar la idea de una recirculación o circuito cerrado también para el material granular. Esto es aún más necesario para estudiar ríos de gran pendiente y transporte. 
Así, se han hecho en destacados experimentos en ríos de grava (Wilcock \& McArdell, 1993; Marion \& Fraccarollo, 1997).

La instalación básica es un canal de $30 \mathrm{~m}$ de longitud, de inclinación variable, con sección rectangular de $0.75 \mathrm{~m}$ de anchura y $0.60 \mathrm{~m}$ de altura, paredes de vidrio y fondo de PVC. Tal canal está alimentado por un circuito de agua compuesto por: 1) un depósito acumulador subterráneo de $1.5 \mathrm{~m}$ de profundidad y $200 \mathrm{~m}^{2}$ de superficie; 2) tres bombas de $90 \mathrm{l} / \mathrm{s}$ cada una; 3) un depósito regulador elevado $\left(20 \mathrm{~m}^{3}\right)$ de carga, y 4) una conexión por medio de tres tuberías flexibles, con sendas válvulas y caudalímetros desde el depósito regulador al canal. La disipación de energía se realiza en la cabecera, en un área un poco más profunda.

El canal de pendiente variable se apoya y sujeta en un depósito de desembocadura (arqueta) al que está articulado (bisagra), mientras que en el extremo de aguas arriba está libre, razón de que las tuberías de entrada sean flexibles. La arqueta de la desembocadura es un recinto grande, con la misión de separar el agua del material granular (Figura 1). Esta función es esencial para que sea viable un circuito de sólidos sin pérdida apreciable de material. Anteriormente se había proyectado un canal que estaba articulado aguas arriba mientras era libre aguas abajo, en donde se prolongaba con una pequeña arqueta solidaria (Martín-Vide \& Andreatta, 2006). En esta pequeña arqueta el flujo era tan agitado que escapaban granos de arena con el agua a partir de cierto caudal. En el caso de este artículo, la arqueta es tan grande que no hay pérdida de granos incluso para el caudal máximo de $100 \mathrm{l} / \mathrm{s}$. La arqueta es un tronco de pirámide invertido para que en su fondo se recoja el material granular. La salida superficial de agua de la arqueta está controlada con una compuerta a la que sigue un vertedero triangular de pared delgada, que sirve de comprobación del caudalímetro en cabecera. 


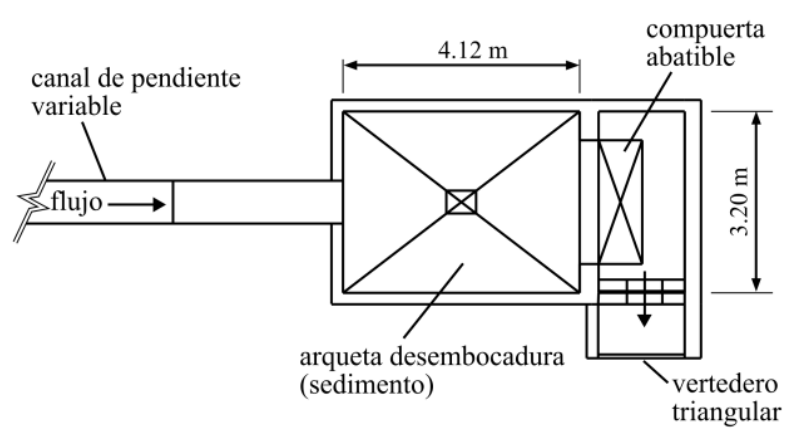

Figura 1. Arqueta de separación de agua y sedimento vista en planta. Para el corte, ver la Figura 2.

El circuito del material granular, más complejo que el del agua, comienza en esta arqueta de desembocadura. Sustituye a una recirculación manual frecuente en muchos experimentos (Wilcock \& McArdell, 1993), pero no es único en la bibliografía (Marion \& Fraccarollo, 1997), aunque sí destaca por sus dimensiones. Una bomba aspira el sedimento con agua desde el fondo de la arqueta y lo impulsa por una tubería de $10 \mathrm{~cm}$ de diámetro hacia unos aparatos en la zona de cabecera, cuyo objetivo (Figura 2) es separar las dos fracciones granulares (arena y grava), escurrirlas y llevarlas a dos destinos posibles: la cabecera del canal o el almacenamiento temporal en silos. La separación básica entre arena y grava se realiza, a la cota más alta, con un aparato originario de la minería, llamado trommel, que consiste en un tambor giratorio, de eje ligeramente inclinado respecto a la horizontal, con la pared revestida de malla, cuyo paso es de $2.25 \mathrm{~mm}$. La tubería de impulsión descarga directamente el chorro de agua con arena y grava en el interior de este tambor. La arena (diámetro $<2.25$ $\mathrm{mm}$ ) y el agua escapan por la malla, pero la grava $(\geq 2.25 \mathrm{~mm}$ ) prácticamente escurrida llega al extremo inferior del tambor sin cruzarla. Esta malla es una "piel", fácil de cambiar por otra de distinto paso si se quiere modificar la cifra de $2.25 \mathrm{~mm}$ que separa la fracción fina de la gruesa. A la arena escapada del tambor le falta ser separada del agua: un desarenador con tornillo sinfín extrae la arena prácticamente escurrida. 


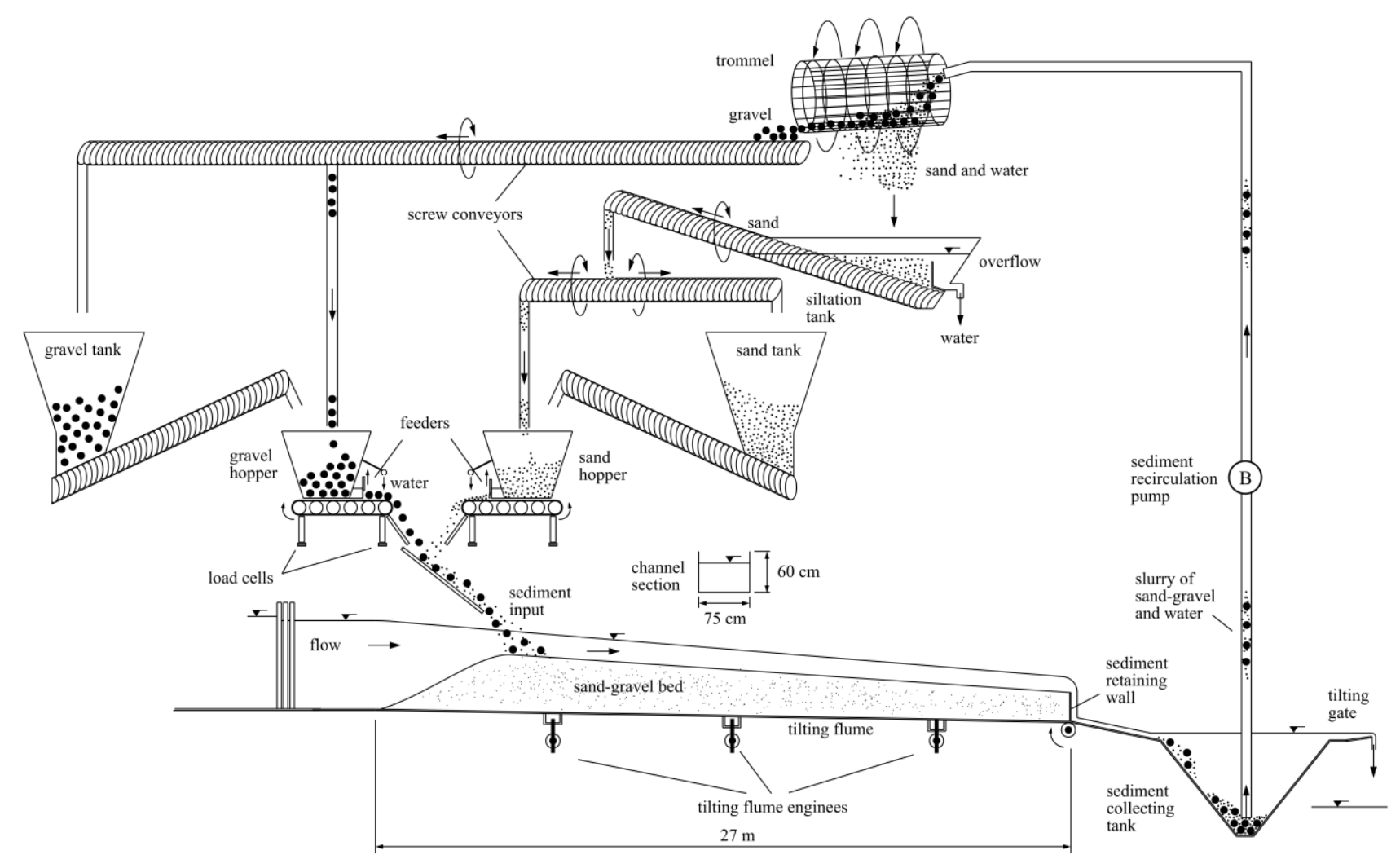

Figura 2. Circuito completo del material granular en la instalación.

La experiencia con esta primera parte del circuito ha sido satisfactoria. El desarenador es el elemento que mejor funciona: tiene gran capacidad de extracción; no se pierde arena por el desagüe; en funcionamiento continuo mantiene constante la carga sólida en su interior y puede trabajar bien con distintos grados de carga; el tambor trommel funciona bien: tan sólo necesita alguna vez un chorro de agua de limpieza para despedir las partículas atoradas en la malla; la bomba ( $y$ en menor medida la tubería) sufre una abrasión considerable por los sólidos, que se ha remediado con un cambio frecuente de rodete; por último, la mayor dificultad es la aspiración del fondo de la arqueta: en ocasiones la boca de aspiración queda enterrada o atorada, y en otras no es 
completamente eficaz en aspirar el material; lo mejor es que la boca tenga libre la orientación de la aspiración.

La segunda parte del circuito del material granular, menos importante, consiste en llevar la arena y la grava a sus dos destinos posibles, el principal —el canal-y el auxiliar —los silos-. El desplazamiento se realiza con tornillos sinfín para ambos materiales. En el caso de la arena, por ejemplo, cambiando el sentido de giro del tornillo, la arena se dirige al silo (almacenamiento) o al canal (recirculación). El caso de la grava es similar. Los dos silos iguales, uno para cada material, tienen un volumen de $6 \mathrm{~m}^{3}$. Los dos están equipados con escotillas de fondo y tornillos sinfín para extraer y transportar el material hacia el canal.

La tercera parte del circuito, de hecho la más importante, tiene el objetivo de suministrar la arena y la grava al canal de forma controlada. Para ello, se cuenta con dos tolvas dosificadoras de acero, una para cada material, con la típica forma de tronco de pirámide invertido y una capacidad de 900 litros cada una. Cada tolva recibe su material por el camino de la recirculación (trommel-desarenador-tornillo sinfín para la arena y trommel-tornillo sinfín para la grava) o desde los silos con tornillos sinfín, en caso de reposición de material. Estas tolvas son necesarias para alimentar un ensayo con un caudal sólido a voluntad. La dosificación de las tolvas se sirve de la apertura de una compuerta plana deslizante y de la velocidad de una cinta extractora.

\section{Medidas y modos de funcionamiento}

Cada tolva dosificadora descansa en cuatro puntos, en donde cuatro células de carga dan continuamente el peso total del aparato incluido su contenido. Con esta medida de peso es posible calibrar las tolvas, es decir conocer el caudal sólido en peso que suministran al canal. Usando la analogía con la hidráulica, el caudal sólido suministrado por la tolva se 
expresaría como área $\times$ velocidad, o sea el producto de la apertura de la compuerta (área $A$, igual a altura por anchura) por la velocidad de la cinta $(v)$. Se han realizado ensayos de calibración de la tolva consistentes en el vaciado de su contenido con una tabla de distintas velocidades de la cinta y aperturas de la compuerta. Así, se ha probado que la cantidad suministrada por las tolvas es constante a lo largo del vaciado, con independencia de su grado de llenado. Esta es una propiedad conocida en la ingeniería de dosificación de granulados. En segundo lugar, el caudal sólido $\left(Q_{s}\right)$ es, en efecto, una función monótona creciente de los dos parámetros: apertura y velocidad, pero está lejos de ser igual a su producto. Durante la calibración se tomaban muestras del material dosificado por cada tolva para determinar su humedad $(\omega)$. También se sabe la densidad $(\rho)$ y el índice de huecos $(\varepsilon)$ de cada material. El cociente de estas magnitudes en la forma adimensional $Q_{s}$ $/[A \cdot v \cdot \rho \cdot(1-\varepsilon) \cdot(1+\omega)]$, notado como $\eta$, expresa hasta qué punto la analogía es válida. Si este coeficiente fuera la unidad, el material granular sería extraído por la cinta exactamente como una materia arrastrada, pero el coeficiente siempre es menor que 1 , más cerca de la unidad para la grava que para la arena. El resultado se da en la Figura 3 (Núñez-González, 2012): para cualquier apertura de compuerta y velocidad de la cinta se obtiene el valor de $Q_{s}$ suministrado por la instalación. Con este caudal sólido calibrado y el caudal de agua (caudalímetros) queda definido un ensayo de lecho móvil.

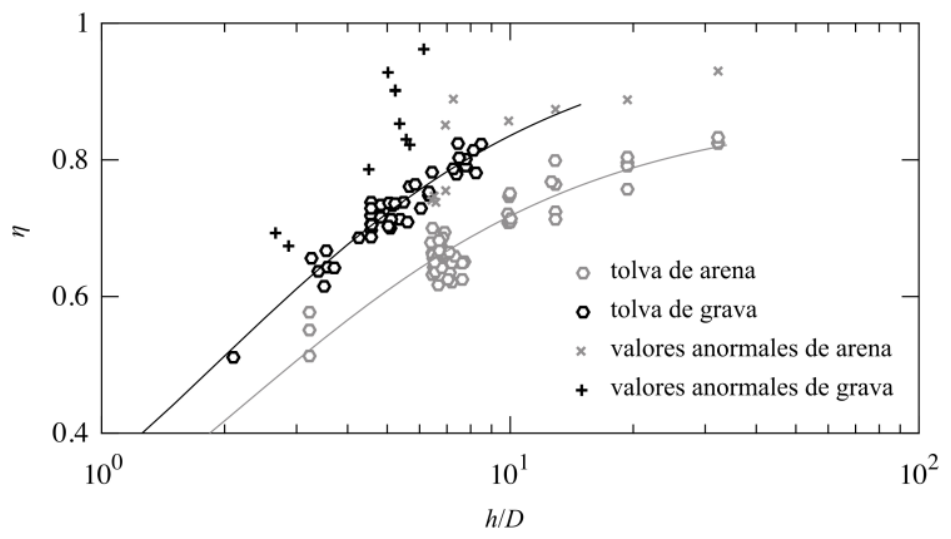

Figura 3. Calibración de las tolvas de arena y grava, en términos del coeficiente adimensional $\eta$ descrito en el texto; $h$ es la apertura (altura) de compuerta, $A=h \cdot B$, 
con $B$ su anchura (constante); $D$ es el tamaño de grano. Los "valores anormales" ocurren con velocidades demasiado bajas de la cinta extractora.

Las tolvas reciben el material recirculado al ritmo irregular de su salida del canal, ritmo quizá más irregular aún por los azares en la impulsión y la separación, y demorándose más o menos en el camino. Pero sólo puede haber cierto almacenamiento temporal en la arqueta de desembocadura (o sea, que esté más llena o más vacía), no en la bomba, ni en la tubería, trommel y desarenador (el cual mantiene carga constante). Estas irregularidades y desfases temporales son precisamente amortiguados y corregidos por las tolvas, pues entregan al canal un caudal de arena y de grava constantes cualquiera que sea su grado de llenado.

La variación temporal del peso de la tolva, registrada de modo continuo, es la diferencia entre el caudal sólido que entra a la tolva y el que sale dosificado. Por tanto, si se cuida que el material no se almacene en el camino (en la arqueta), la variación del peso de la tolva indica la diferencia entre la capacidad de transporte del canal y el caudal sólido suministrado al canal. Si el peso de la tolva va aumentando, es necesariamente porque el volumen aluvial en el canal está disminuyendo (erosión del lecho), debido a que la capacidad de transporte es mayor que el suministro; si, en cambio, el peso va disminuyendo, es porque el volumen está aumentando (acreción del lecho), debido a que el suministro es mayor que la capacidad; si el peso es constante, es porque hay equilibrio, es decir, el suministro y la capacidad coinciden. Un peso de las tolvas constante es, por tanto, la mejor medida del equilibrio del ensayo.

También se puede hacer otro tipo de ensayo de recirculación, llamado pura, si las tolvas están vacías, sus compuertas abiertas y la cinta extractora en marcha, de modo que la arena y la grava devueltas a cabecera crucen cada tolva vacía en un instante, casi como si no existieran. Se puede también hacer un ensayo de alimentación pura, en la que el material suministrado no es material recirculado sino aportado desde los silos, manteniendo también vacías las tolvas. En el modo de funcionamiento habitual, llamado "recirculación", la medida en las tolvas (peso vacío + peso del contenido) sirve para estudiar el equilibrio. Este 
modo se caracteriza porque las tolvas entregan el material como en alimentación, como si fueran silos más pequeños, pero el material ha circulado ya por el canal, más exactamente acaba de circular por él. En rigor, se trata de un híbrido entre recirculación y alimentación. El material en efecto es recirculado, pero se suministra de nuevo al canal con una tasa controlada de forma independiente por las tolvas, como en un ensayo de alimentación.

\section{Ejemplos de funcionamiento}

Para ilustrar los tipos de ensayo y discutir su significado se presentan a continuación cuatro ejemplos (Núñez-González, 2012). La Figura 4 muestra el peso en las dos tolvas a lo largo del tiempo en un ensayo de recirculación (modo habitual). El lecho inicial está formado por una mezcla de arena y grava fina, confinada en el extremo de aguas abajo por una barrera transversal a ras de lecho (siempre presente, de lo contrario el lecho se desmoronaría). Ambas tolvas comienzan el ensayo relativamente vacías, suministrando desde el inicio un caudal sólido constante de $0.137 \mathrm{~kg} / \mathrm{s}$, compuesto por los mismos porcentajes de arena y grava (32 y 68\%) que se encuentran en el lecho. En un primer momento las tolvas se vacían todavía más, pero pronto empiezan a recibir el material recirculado, llenándose poco a poco. Los tramos cuasihorizontales de ambas líneas a partir de las $7 \mathrm{~h}$ indican la aproximación al equilibrio. El aumento de peso en las tolvas es prueba de que al equilibrio se ha llegado en un proceso de incisión del lecho debido a que el caudal sólido era escaso para la capacidad de transporte, dado el caudal $(78.4 \mathrm{l} / \mathrm{s})$, la pendiente inicial del fondo $(0.86 \%)$ y la granulometría. 

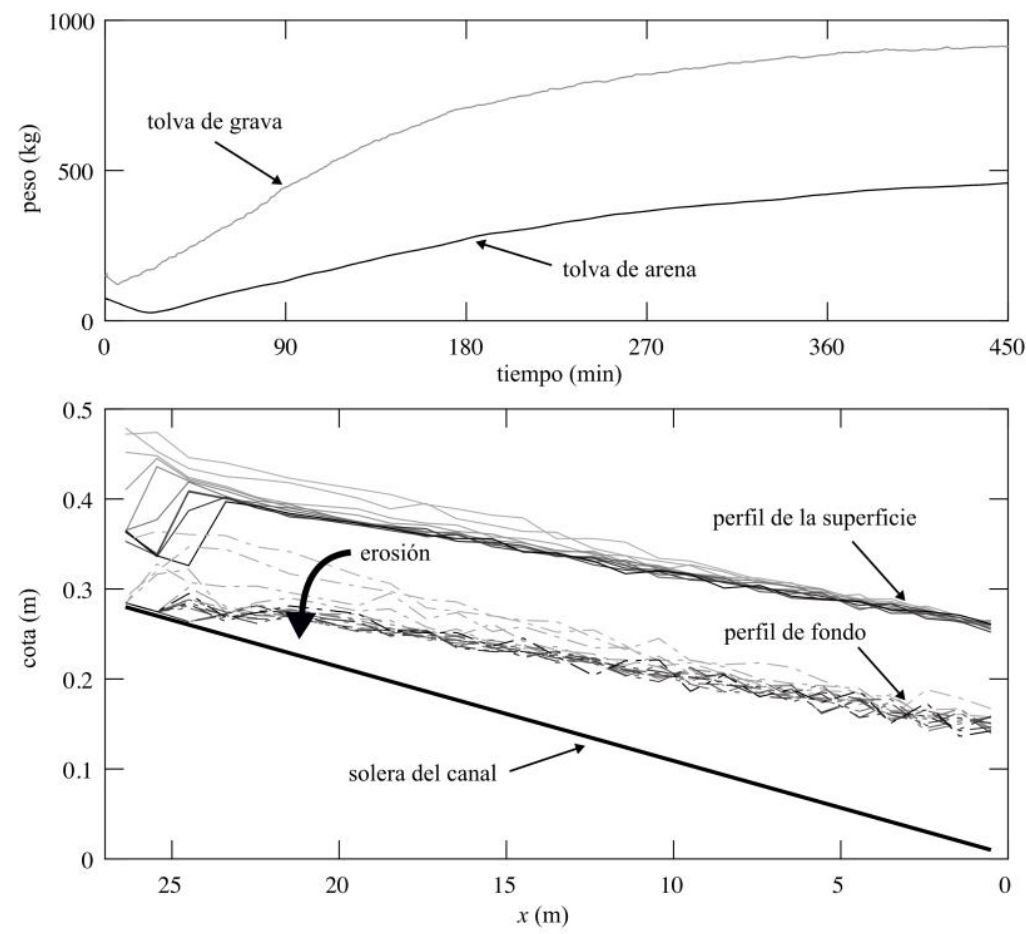

Figura 4. Ensayo de una mezcla de arena $(1.4 \mathrm{~mm}, 32 \%)$ y grava $(4.1 \mathrm{~mm}, 68 \%)$, bajo un caudal sólido de $0.137 \mathrm{~kg} / \mathrm{s}$, líquido de $78.4 \mathrm{l} / \mathrm{s}$. Arriba: peso en ambas tolvas.

Abajo: perfiles del fondo y del agua cada hora (flujo de izquierda a derecha).

Otro punto de interés de la Figura 4 es el diferente tiempo de recuperación de peso de una y otra tolva. Al empezar el ensayo, la arena recirculada se demora en llegar unos 20 minutos, unas tres veces más de lo que tarda en llegar la grava. La razón es el recorrido más largo de la arena, por la presencia del desarenador en su circuito (Figura 2 ). No obstante, a largo plazo, las ganancias de peso en una y otra tolva deben guardar la misma proporción que la composición del lecho que se va erosionando si la granulometría del material en movimiento es la misma que la del material del fondo, como es el caso de este ejemplo.

El modo en que en efecto ha ido ocurriendo la incisión se puede ver en la Figura 4. Al perfil de fondo inicial de más pendiente le sucede un perfil final de menos pendiente, que ha girado respecto al extremo de aguas abajo, donde se encuentra la barrera del lecho $(x=0)$. Para estudiar este cambio de pendiente se han ajustado rectas a los datos Tecnología y ciencias del agua, 10(2), DOI: 10.24850/j-tyca-2019-02-10 

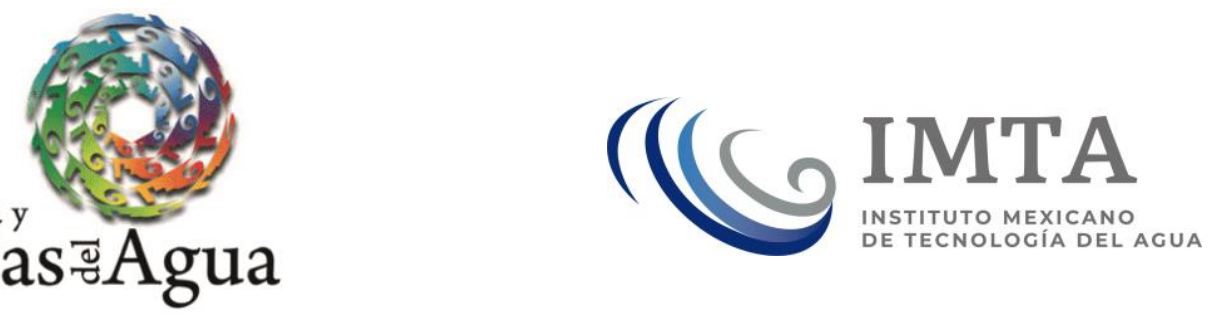

experimentales, al inicio y al final del ensayo, excluyendo los primeros $10 \mathrm{~m}$ de canal afectados por la aceleración del agua y la curvatura del fondo. La pendiente del agua va disminuyendo al unísono de la baja de la pendiente del fondo. El régimen es más o menos uniforme en los tramos ajustados, de más de $15 \mathrm{~m}$ de longitud, aunque en rigor sólo es uniforme en el estado de equilibrio final.

El segundo ejemplo es también de recirculación en el modo habitual, en este caso con lecho de grava y con un caudal sólido de suministro $(0.081 \mathrm{~kg} / \mathrm{s}$ de la misma grava) superior a la capacidad del transporte sólido en el canal. En consecuencia, la tolva de grava, que comienza con menos de $400 \mathrm{~kg}$, se va vaciando (Figura 5). El riesgo de quedar vacía llevó a una reposición con grava del silo tres veces (produciendo los saltos verticales de peso en la Figura 5). En otro momento se empleó el artificio de desviar hacia los silos el material recirculado para conocer el caudal suministrado en realidad por la tolva sin recurrir a la calibración de la Figura 3. Esto se manifiesta en la línea de máxima pendiente en descenso (Figura 5). El cambio de perfil de fondo y superficie libre muestra el comportamiento opuesto a la incisión del primer ejemplo: una acreción, debida a un suministro mayor que la capacidad de transporte. La pendiente aumenta de forma ligera, ya que el perfil del fondo gira como antes respecto $a x=0$. 


\section{Tecnología y}
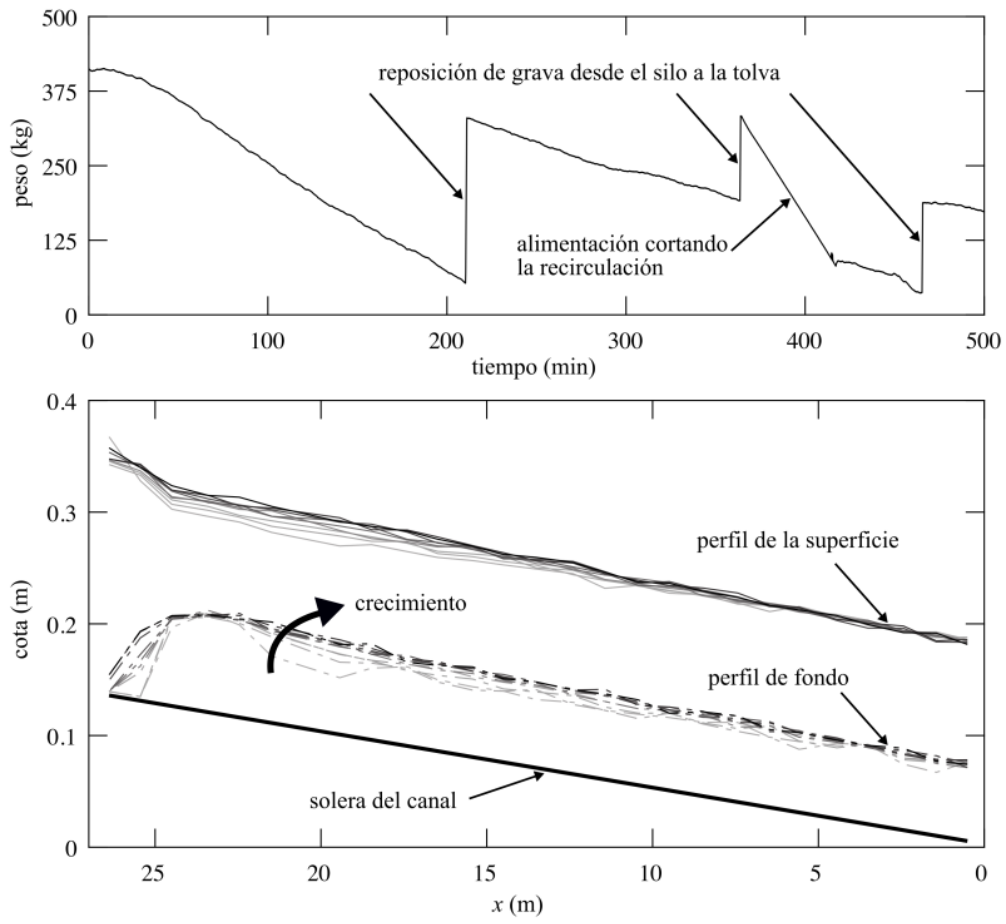

Figura 5. Ensayo de un lecho de grava $(4.1 \mathrm{~mm}, 100 \%)$, con caudales de $78.7 \mathrm{l} / \mathrm{s}$ y sólido de $0.081 \mathrm{~kg} / \mathrm{s}$ en el canal. Arriba: peso en la tolva. Abajo: perfiles del fondo y del agua, cada hora. La pendiente de la solera del canal es $0.5 \%$.

El tercer ejemplo es de recirculación pura. Un lecho semejante al del primer ejemplo (32\% de arena, $68 \%$ de grava) con pendiente inicial del fondo $1.28 \%$ se somete al flujo de $78.4 \mathrm{l} / \mathrm{s}$ y a la recirculación pura, como si no existieran las tolvas. Si la capacidad de transporte es muy elevada, como es el caso con una pendiente tan alta, el caudal sólido recirculado será al poco tiempo también muy elevado, tanto como para igualar a la capacidad, y lo mismo pasaría con valores menores de capacidad de transporte. Así, en este tipo de ensayos, los cambios de pendiente y régimen hidráulico con respecto al estado inicial pueden ser de poca importancia (por ello no se presentan perfiles de fondo y agua en la Figura 6). Ésta es una propiedad de la recirculación pura en la instalación que se presenta. 


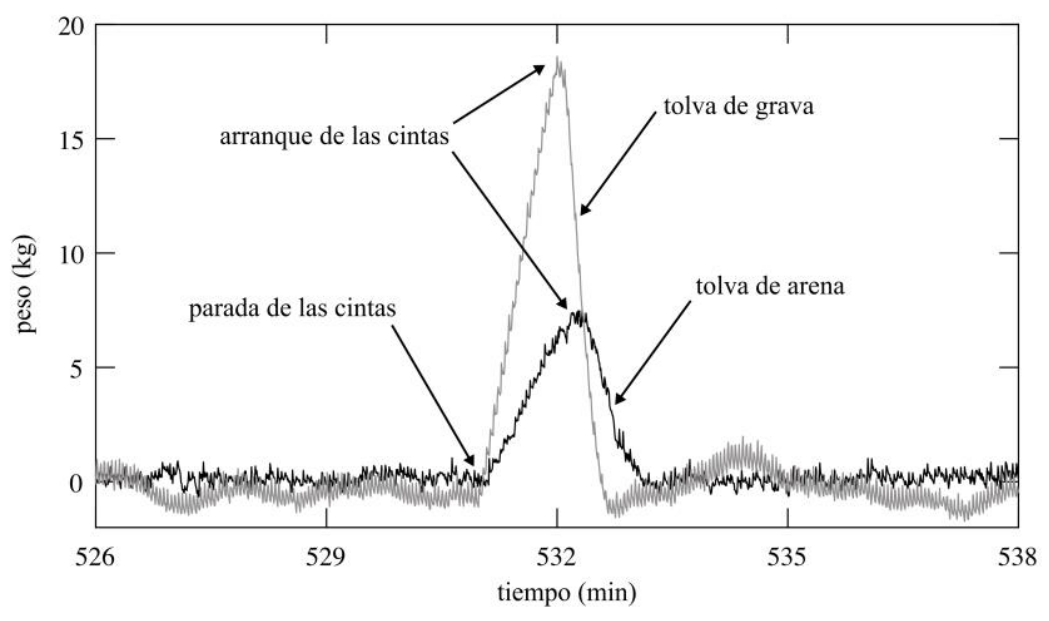

Figura 6. Peso en las tolvas (vacías) de arena y grava, incluyendo 1 minuto de interrupción de la dosificación, en un ensayo de recirculación pura.

Dado que el peso de las tolvas es siempre nulo, parece que se perdería la posibilidad de medida del caudal sólido. No obstante, la medida se puede hacer parando de modo momentáneo las cintas extractoras y determinando la pendiente con que crece el peso en las tolvas por acumulación del material recirculado. Este artificio se puede aplicar en cualquier otro ensayo para conocer en un momento dado el caudal sólido recirculado. Al volver a arrancar las cintas tras la interrupción, el volumen acumulado se entrega en seguida al canal. La operación se ilustra en la Figura 6 con una interrupción de un minuto, tiempo corto para no alterar el ensayo. El peso de las tolvas es de alrededor de cero con la excepción del minuto de medida, en el que resulta $0.300 \mathrm{~kg} / \mathrm{s}$ de grava y $0.133 \mathrm{~kg} / \mathrm{s}$ de arena, proporción muy semejante a la composición del lecho. La cifra total de $0.433 \mathrm{~kg} / \mathrm{s}$ es la más alta de los cuatro ejemplos. El máximo caudal sólido ensayado en la instalación ha sido $1.07 \mathrm{~kg} / \mathrm{s}$ y la máxima pendiente $2.14 \%$.

El cuarto ejemplo es un caso particular de alimentación pura, en concreto, el de un caudal sólido de alimentación nulo (las dos tolvas tienen la cinta extractora parada y la compuerta cerrada). El lecho es una mezcla de la misma arena y grava, aunque en proporciones distintas: $40 \%$ de arena y $60 \%$ de grava. La Figura 7 muestra el rápido crecimiento del peso en las tolvas debido a la erosión del lecho. El perfil 
de fondo gira respecto a $x=0$ y la superficie libre acompaña al cambio de pendiente del fondo (Figura 7). El ensayo no ha alcanzado ni mucho menos el equilibrio después de $6 \mathrm{~h}$, pues no habrá equilibrio hasta que la capacidad de transporte en el canal sea cero, pues el suministro de material es nulo. La pendiente inicial del lecho es $0.75 \%$; al cabo de $6 \mathrm{~h}$ es $0.29 \%$. El tiempo que tarda cada tolva en empezar a recibir el material erosionado es parecido al tiempo de reacción del ejemplo primero (la arena responde de manera más lenta que la grava, pues el camino es el mismo). La proporción entre las dos curvas de crecimiento es semejante a la proporción entre grava y arena en la composición del lecho.
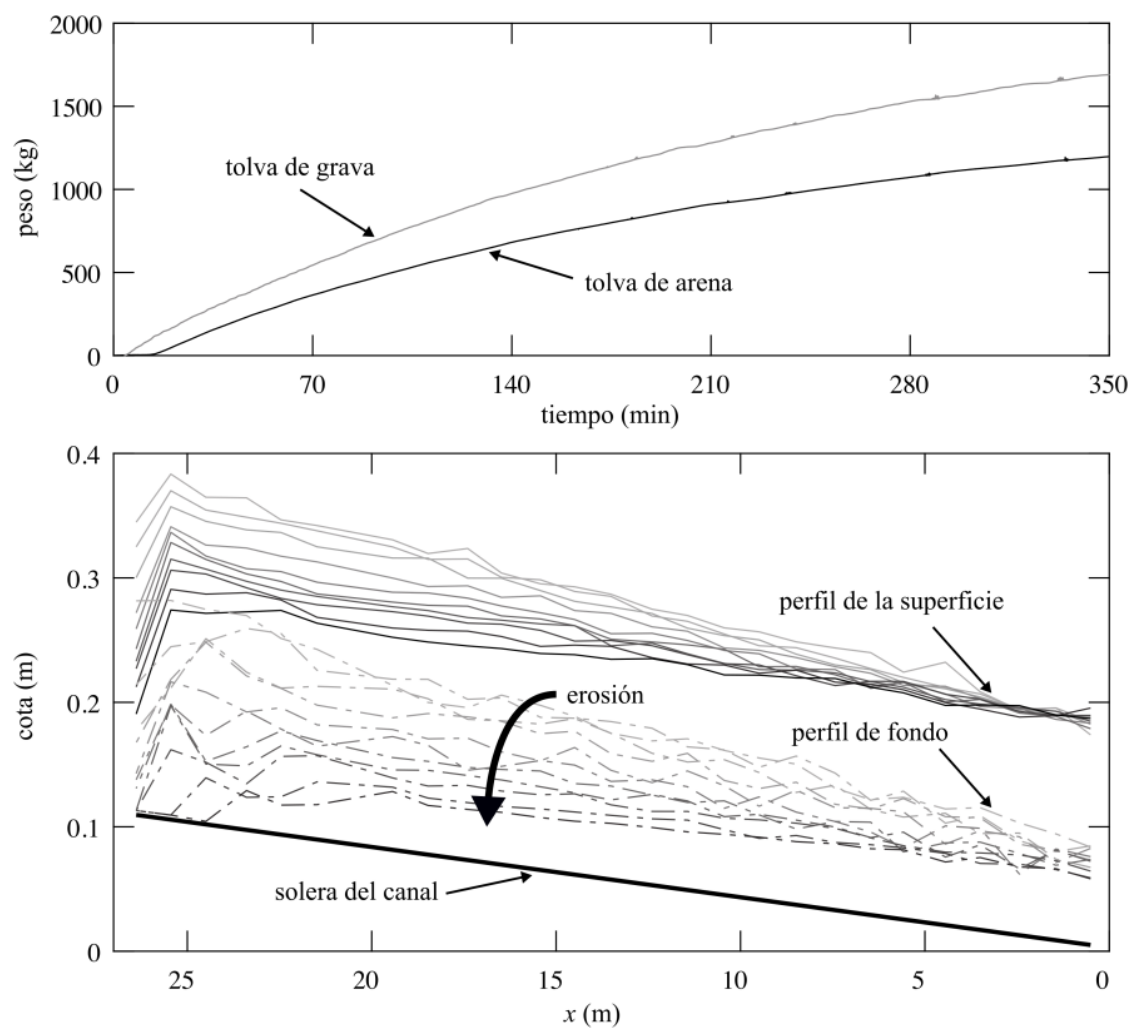

Figura 7. Ensayo de un lecho de arena (1.4 mm, 40\%) y grava $(4.1 \mathrm{~mm}, 60 \%)$, caudal de $78.9 \mathrm{l} / \mathrm{s}$ y caudal sólido nulo. Arriba: peso en las tolvas. Abajo: perfiles del fondo y el agua en el canal, cada hora. Pendiente de la solera del canal $0.40 \%$. 

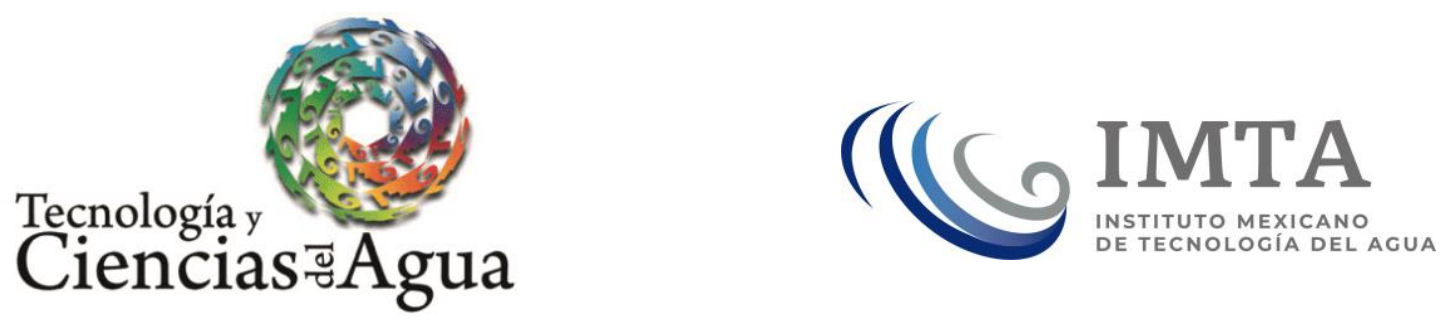

\section{Discusión}

En este apartado se discute cómo relacionar tales ensayos en canal de laboratorio con tramos fluviales reales. La semejanza del lecho móvil en el canal con el prototipo de un río impone respetar algunos criterios, por ejemplo el número de Reynolds debe estar dentro del intervalo del régimen turbulento rugoso. Ahora bien, otros ensayos que estudian procesos (como el transporte sólido) son de hecho "sin prototipo". En todos los casos, un experimento grande, en cuanto al caudal, calado, caudal sólido $y$, por tanto, velocidad y turbulencia, aproxima el laboratorio a la realidad. El canal descrito es casi un río dentro del laboratorio si se toma en cuenta el caudal unitario máximo de $270 \mathrm{l} / \mathrm{s}$ $/ 0.75 \mathrm{~m}=0.36 \mathrm{~m}^{2} / \mathrm{s}$ y el caudal sólido unitario máximo $1.07 \mathrm{~kg} / \mathrm{s} / 0.75$ $\mathrm{m}=1.43 \mathrm{~kg} / \mathrm{s} / \mathrm{m}$.

La correspondencia con un río real se enfrenta en primer lugar a la pregunta de cómo un segmento representa el río continuo. Al cortar el río por una sección, identificada con el contorno aguas arriba de un canal, hay que representar el resto del río por su caudal líquido y sólido. Pero en la práctica el canal no puede trabajar sin recirculación, lo cual introduce una dificultad conceptual en la interpretación de los ensayos. Se supone que se ha dado una pendiente al lecho móvil del canal y se ensaya un caudal de agua. El caudal sólido resultante que sale del canal es igual a la capacidad de transporte con esa pendiente. $\mathrm{Si}$, a continuación, se devuelve este caudal sólido a la cabecera en "recirculación pura", se estará alimentando el tramo con su propia capacidad. La recirculación significa entonces que un tramo imaginario aguas arriba, el que alimentaría al canal con cierto caudal sólido, sería exactamente igual al propio canal. En consecuencia, el río que se pretende simular en el laboratorio es idéntico a sí mismo hacia arriba en una longitud ilimitada. No es raro por ello que los cambios con respecto 
al estadio inicial sean mínimos. Esto es una simplificación de la realidad, porque los ríos no son iguales a sí mismos al desplazarnos hacia arriba, sino que todas sus variables van cambiando: caudal, anchura y profundidad, granulometría, etcétera.

$\mathrm{Si}$, en cambio, se establece un caudal sólido con las tolvas ("alimentación pura"), quedará determinada de forma unívoca una pendiente de equilibrio en el canal, dado el caudal de agua y el tamaño de grano. Este caudal sólido debería ser en realidad el que el río aporte al tramo desde aguas arriba, pero sobre este suministro de sólidos, a falta de medidas reales, sólo se pueden hacer hipótesis. Si se suministra un caudal sólido alto se causa una pendiente de equilibrio mayor, con acreción; si se alimenta con uno bajo, una pendiente menor, con incisión. ¿Qué significa entonces estudiar el equilibrio si la cantidad con que se alimente lo determina de modo arbitrario? La indeterminación del caudal sólido de alimentación del canal no se resuelve aplicando una fórmula de transporte sólido (¿es válida para el río que estudiamos?), sino sólo con medidas. No se resolvería tampoco, en rigor, enlazando otro tramo de canal aguas arriba del existente. Si bien esto último es irrealizable en el laboratorio, es la manera de razonar y actuar con un modelo numérico. Lo que se consigue alargando el tramo de estudio aguas arriba es tan sólo desplazar en la misma medida la indeterminación (la arbitrariedad); si bien la sensibilidad de la alimentación en cuestión va disminuyendo al alargar el tramo aguas arriba.

Lo que se acaba de plantear es una pregunta clásica de la hidráulica fluvial. Dado un caudal de agua sobre un fondo aluvial de cierto tamaño de grano, es posible pensar que el caudal sólido es resultado de la pendiente del fondo o, a la inversa, que la pendiente resulta del caudal sólido. ¿Cuál de las dos es la variable independiente y, en consecuencia, cuál la dependiente?; en el caso que se presenta en este trabajo, ¿cuál de las dos variables se debe imponer en un ensayo? La propiedad esencial del equilibrio (el perfil del fondo estable) ayuda en esta dificultad, pues cuando se alcanza un equilibrio es seguro que el caudal sólido de alimentación recirculando es igual a la capacidad de transporte del canal, manifestada como caudal sólido que sale. Entra y sale el mismo caudal sólido, o de lo contrario, por conservación de masa, no 
estaría el fondo en equilibrio. La indeterminación del párrafo anterior se convierte por lo tanto en discrecionalidad, ya que habrá tantos equilibrios distintos como se quiera. En todos ellos, la alimentación sólida será igual a la capacidad de transporte del canal; en todos se espera alcanzar un régimen uniforme y una cierta pendiente de equilibrio; en todos, la alimentación determinará de forma unívoca la pendiente. Todos serán ensayos valiosos, pero no se sabrá cuál corresponde al río que se desea estudiar. De alguna manera, el ensayo en canal se desvincula del prototipo, no se puede anclar a él sin más información.

Una dificultad más surge cuando se reconoce que el transporte real de un río puede estar determinado no por su capacidad de transporte sino por la "disponibilidad" de material. Por ello, sobre el uso de fórmulas de transporte sólido para alimentar ensayos representativos de un río caben no una sino dos preguntas: además de ¿es válida la fórmula para el río?, hay que preguntarse ¿el transporte del río es igual a su capacidad o está controlado por la disponibilidad? La noción de disponibilidad refuerza que una alimentación verdadera es esencial, o sea refuerza el caudal sólido como variable independiente.

Como se ha dicho, la instalación combina recirculación con alimentación. Una particularidad de este modo híbrido de la instalación tiene que ver con la granulometría de los materiales. Si una fracción del material del lecho no fuera movida por el agua, o fuera movida en menor medida que su presencia en el lecho, contribuyendo así menos al transporte sólido, el caudal parcial de esa fracción salido del canal sería menor (Wilcock \& McArdell, 1993), y así su presencia en la tolva correspondiente también iría siendo menor. Esto es más probable con los tamaños más gruesos y se llama "transporte parcial o selectivo". De manera inversa, esta fracción gruesa menos móvil participaría en el acorazamiento del lecho. En tal caso, los estados de equilibrio alcanzados en recirculación pura y en alimentación pura serían diferentes (Parker \& Wilcock, 1993), pues lo recirculado tiene la composición de lo salido del canal, pero lo alimentado tiene siempre la misma proporción, igual a la del lecho inicial. En experimentos en que el agua mueve todas las fracciones de la mezcla del lecho casi por igual, 
no de forma selectiva ("movilidad igualada"; Parker and Klingeman, 1982); esta diferencia en el equilibrio sería despreciable.

Con lo que se acaba de señalar, el modo híbrido de la instalación no se distingue de una alimentación pura, en la que el material viene de modo directo de los silos según cantidades constantes si cada tolva dosifica de manera invariable, prestablecida, a pesar de que su contenido vaya disminuyendo (o aumentando) durante el ensayo. Ahora bien, si la dosificación tiene en cuenta la variación del contenido, buscando que cada fracción alcance un peso constante, entonces el modo híbrido se diferencia de la alimentación pura. Esta posibilidad de regulación de la tolva hace que la instalación sea propiamente híbrida.

En muchos ensayos semejantes a los presentados no se ha observado en ningún caso un descenso del peso en la tolva de grava, compensado, para tener peso total constante, como exige el equilibrio, por un ascenso del peso en la tolva de arena, lo cual habría sido prueba de un transporte selectivo. Al buscar equilibrios con pendientes y cargas sólidas elevadas, pero siendo la arena bastante gruesa $(1.4 \mathrm{~mm})$ y la grava bastante fina $(4.1 \mathrm{~mm})$, se puede decir que se ha trabajado siempre en condiciones de movilidad igualada. Gracias a la medida del transporte de arenas y gravas en dos tolvas diferentes, la instalación es indicada para estudios de transporte parcial o selectivo. Precisamente, los cambios de peso de las tolvas servirían para medirlo. Del mismo modo, se distinguiría un proceso de acorazamiento de la superficie del lecho, si se diera.

\section{Conclusión}

Se ha descrito una instalación para estudios de equilibrio fluvial con lechos de arena y grava. Es singular por sus grandes dimensiones, en particular por ser capaz de mantener ensayos de gran duración en que se transporte incluso $1 \mathrm{~kg} / \mathrm{s}$. La recirculación es imperativa para Tecnología y ciencias del agua, 10(2), DOI: 10.24850/j-tyca-2019-02-10 
manejar un ensayo con gran volumen de material granular, pero está indeterminado cuál debe ser el caudal de alimentación. Sólo medidas reales resuelven la indeterminación. La solución de que sea igual a la capacidad de transporte significa una simplificación de la realidad (el río no es uniforme aguas arriba). Pero esta identificación entre alimentación y capacidad es inherente al estudio del equilibrio y sirve para combatir la indeterminación, si bien al precio de volver arbitrario el ensayo. La instalación descrita gracias a su control independiente del transporte de arena y grava es indicada para el estudio del transporte parcial o selectivo. Los ejemplos explicados, tanto de erosión como de acreción, en modo de recirculación y de alimentación, han tenido en común ser ensayos de movilidad igualada.

\section{Nota}

La instalación se encuentra en la Universidad de Castilla-La Mancha, España. Los autores se encargaron de proyectarla y ponerla en marcha. El segundo autor desarrolló en ella los experimentos de su tesis doctoral (Núñez-González, 2012).

\section{Referencias}

Marion, A., \& Fraccarollo, L. (1997). Experimental investigation of mobile armoring development. Water Resources Research, 33(6), 1447-1453.

Martín-Vide, J. P., \& Andreatta A. (November, 2006). Disturbance caused by bed sills on the slope of steep streams. Journal of Hydraulic Engineering ASCE, 132(11), 1186-1194.

Núñez-González, F. (junio, 2012). Bedload transport of sand-gravel mixtures with Antidunes. Flume experiments (tesis doctoral). Barcelona, Universitat Politècnica de Catalunya. 
Parker, G. (2008). Transport of gravel and sediment mixtures. En García, M. H. (ed.). Sedimentation engineering. Virginia, USA: ASCE, Reston.

Parker, G., \& Klingeman, P.C. (1982). On why gravel bed streams are paved. Water Resources Research, 18(5), 1409-1423.

Parker, G., \& Wilcock, P. R. (1993). Sediment feed and recirculating flumes: Fundamental difference. Journal of Hydraulic Engineering ASCE, 119(11), 1192-1204.

Wilcock, P. R., \& McArdell, B. W. (1993). Surface-based fractional transport rates: Mobilization thresholds and partial transport of a sand gravel sediment, Water Resources Research, 29(4), 12971312. 